\title{
Is Sociality Driven by the Costs of Dispersal or the Benefits of Philopatry? A Role for Kin-Discrimination Mechanisms
}

\author{
Nicolas Perrin ${ }^{\star}$ and Laurent Lehmann
}

Institute of Ecology, University of Lausanne, CH-1015 Lausanne, Switzerland

Submitted September 19, 2000; Accepted May 30, 2001

\begin{abstract}
The role of ecological constraints in promoting sociality is currently much debated. Using a direct-fitness approach, we show this role to depend on the kin-discrimination mechanisms underlying social interactions. Altruism cannot evolve under spatially based discrimination, unless ecological constraints prevent complete dispersal. Increasing constraints enhances both the proportion of philopatric (and thereby altruistic) individuals and the level of altruistic investments conceded in pairwise interactions. Familiarity-based discrimination, by contrast, allows philopatry and altruism to evolve at significant levels even in the absence of ecological constraints. Increasing constraints further enhances the proportion of philopatric (and thereby altruistic) individuals but not the level of altruism conceded. Ecological constraints are thus more likely to affect social evolution in species in which restricted cognitive abilities, large group size, and/or limited period of associative learning force investments to be made on the basis of spatial cues.
\end{abstract}

Keywords: altruism, associative learning, game theory, kin competition, relatedness, spatially based discrimination.

The emergence of sociality is not a simple process. We know since Hamilton (1964) that altruistic interactions, in which an actor sacrifices some of its individual fitness to the benefit of a recipient, must take place among kin (altruism is here defined sensu stricto, thus excluding social interactions, like "reciprocal altruism," which increase the actor's individual fitness). A necessary first step is thus the building of kin structures through the retention of grown offspring within the parental immediate neighborhood (Brown 1974; Gaston 1978; Emlen 1982). But we also know that kin competition is a powerful force, strong enough to drive complete dispersal if unopposed (Ham-

\footnotetext{
* E-mail: nicolas.perrin@ie-zea.unil.ch.
}

Am. Nat. 2001. Vol. 158, pp. 471-483. (c) 2001 by The University of Chicago. 0003-0147/2001/15805-0002\$03.00. All rights reserved. ilton and May 1977; Frank 1986; Taylor 1988). It follows that altruism cannot evolve unless a selective force opposes kin competition, preventing it from driving complete dispersal, and thereby allowing kin structures to emerge. The nature of this force remains a topic of vivid controversy: "The origin of natal philopatry is the most fundamental problem in cooperative breeding" (Stacey and Ligon 1991, p. 833).

Two lines of thought have emerged, providing seemingly opposite answers to this question (Koenig et al. 1992; Emlen 1994). The first one (e.g., Koenig and Pitelka 1981; Emlen 1982) puts a premium on the ecological constraints limiting the likelihood that a dispersing individual will successfully establish and reproduce in a new territory. These include dispersal costs, habitat saturation, environmental harshness or unpredictability, and any other factor that makes dispersal unlikely to result in successful reproduction. By contrast, the second line of thought (e.g., Zack 1990; Stacey and Ligon 1991; Komdeur 1992) puts a premium on the benefits that potential dispersers may gain by staying home. These may include inheritance of high-quality territory, group-size effects, social interactions with kin, and any other factor that makes philopatry more likely to result in a net fitness gain (be it individual or inclusive).

The point has been made that these two lines should not be seen as alternatives but as complementary hypotheses (e.g., Koenig et al. 1992; Emlen 1994). Indeed, both costs and benefits affect evolutionarily stable strategies, and no one factor by itself causes restricted dispersal and cooperative breeding in general. It remains true, however, that a precise delineation of the relative importance of intrinsic benefits versus extrinsic constraints in molding species-specific patterns is of prime importance in a comprehensive study of social lifestyles. Extrinsic constraints apparently play a crucial role under certain circumstances (e.g., Jarvis et al. 1994; Spinks et al. 2000), while intrinsic benefits seem to predominate in others (e.g., Zack and Ligon 1985; Stacey and Ligon 1987, 1991). Investigating which factors are likely to confer primacy to one set of causes becomes, then, a central question in the study of social systems. This article evaluates the possibility that 
the kin-discrimination mechanisms underlying social interactions might be one of these factors.

Kin discrimination is actually as central to the evolution of sociality as is philopatry. Hamilton (1964) initially suggested that population viscosity might be a sufficient condition for the emergence of altruism. If dispersal is low, neighbors have a higher probability of being relatives than do individuals randomly sampled from the whole population. Thus, interactions among neighbors also occur among relatives by the same token. However, numerical simulations by Wilson et al. (1992) showed that population viscosity might be unable to favor indiscriminate altruism (i.e., randomly directed toward either resident or immigrant neighbors), a result soon confirmed analytically by Taylor (1992). The point is that as long as the habitat is locally saturated neighbors are also more likely to be competitors (the problem obviously differs in unsaturated environments; Mittledorf and Wilson 2000; Ferrière and Le Gaillard 2001). There is no advantage for a focal individual to help neighbors producing more offspring, if these offspring then compete with its own for locally limiting breeding resources. It turns out that the inclusive-fitness benefits gained from indiscriminately helping neighbors exactly cancel out with increased kin-competition costs. As pointed out by Queller $(1992,1994)$, altruism cannot emerge unless social and economic neighborhoods differ: partners must be more related in altruistic than in competitive interactions.

Several kin-recognition mechanisms exist (Waldman 1987; Komdeur and Hatchwell 1999). Which ones are sufficiently reliable to permit the evolution of cooperative breeding in those species where kin selection is an important factor remains an open and crucial question (Komdeur and Hatchwell 1999). Current evidence suggests that familiarity-based recognition, gained through associative learning, is the most likely mechanism enabling helpers to discriminate kin from nonkin in avian societies (Komdeur and Hatchwell 1999). Throughout the period of association that precedes dispersal, the individual characteristics of relatives (e.g., full sibs sharing the same nest or burrow) are memorized and will be remembered after the dispersal period has occurred. Thereby the focal individual will be able to individually recognize residents from immigrants and behave accordingly. In that case, altruistic interactions are highly likely to be directed toward relatives only (and competitive interactions toward nonrelatives), so that the inclusive benefits of sociality (the "benefits of philopatry") might well be strong enough to counteract kin competition and refrain dispersal.

Queller (1992) suggested that a more rudimentary, spatially based discrimination might succeed as well in fostering altruism. As compared to familiarity, a spatially based discrimination does not require sophisticated cognitive abilities: any conspecific met within the natal patch, even after dispersal has occurred, is accepted as a valuable recipient of altruism (no need, therefore, to memorize individual characteristics). It is also obviously much more error prone since unrelated immigrants are as likely as related residents to benefit from it. But, in principle, it may still allow some altruism to evolve: since immigrants have no or few relatives in their new habitat, residents necessarily have a higherthan-average relatedness with their average patch mates (as long as dispersal is not complete). Altruistic interactions (expressed by residents only) are, therefore, statistically more often directed toward relatives than are competitive interactions (expressed by both residents and immigrants).

However, whether the social benefits induced by spatial discrimination are high enough to counteract kin competition and prevent complete dispersal remains an open question. Indeed, a spatially based altruism provides more advantages to immigrants than to residents since the former benefits from it without having to pay its costs. This is bound to bring strong incentives to dispersal, in such a way that the benefits of social interactions may, paradoxically, conjugate with kin competition to drive complete dispersal. Such a process would obviously counterselect any form of sociality.

In this article, we investigate the effects of dispersal costs (meant here to represent any ecological constraint decreasing the probability that a disperser reproduces successfully) on the coevolution between altruism and dispersal, under the two kin-recognition mechanisms delineated above. We show that a spatially based discrimination does allow some altruism to evolve but at a low level and only insofar as dispersal costs prevent complete dispersal. Ecological constraints thus appear as a prerequisite for the emergence of sociality, in strong contrast with what happens under familiarity-based discrimination. Thereby, a formal link is established between the kin-discrimination mechanism underlying a social interaction and the question of whether this interaction evolved through intrinsic benefits or extrinsic constraints.

\section{The Model}

Since optimal strategies for social actions depend on how partners (including relatives) behave, we follow a gametheoretical, kin-selection approach. We use the directfitness method elaborated by Frank and Taylor (Taylor and Frank 1996; Frank 1997, 1998). A gene coding for an action with some social consequences (be it altruism or dispersal) affects the fitness of both actors and social partners. Altruistic interactions, for instance, as already mentioned, bring costs to the actor and benefits to the recipient. The fitness $W_{i j}$ of a focal individual $i$ expressing the gene in its neighborhood $j$ is thus affected by both the direct effect $\left(z_{i j}\right)$ of this gene on himself and the indirect effect $\left(z_{j}\right)$ of 
identical-by-descent copies of this gene found with probability $r$ in related neighbors that interact with him. Hence,

$$
W_{i j}=W\left(z_{i j}, z_{j}\right)
$$

The selective pressure on this gene must account for both effects:

$$
\frac{d W}{d \zeta}=\frac{\partial W}{\partial z_{i j}} \times \frac{d z_{i j}}{d \zeta}+\frac{\partial W}{\partial z_{j}} \times \frac{d z_{j}}{d \zeta},
$$

where $\zeta$ is the breeding value (additive-genetic component of the trait value) of the focal individual (assumed here to be in control of its own actions). The expressions $d z_{i j} / d \zeta$ and $d z_{j} / d \zeta$ can be interpreted as the regression coefficients of the phenotypes of, respectively, the focal individual and its social partner on the focal individual's breeding value (Taylor and Frank 1996). The evolutionarily stable strategy (ESS) is found by setting the selective pressure to zero:

$$
-\frac{\partial W}{\partial z_{i j}}=r \frac{\partial W}{\partial z_{j}},
$$

where

$$
r=\frac{d z_{j}}{d \zeta} \times \frac{d \zeta}{d z_{i j}}
$$

is the kin-selection coefficient of relatedness between actor and recipient (Frank 1998).

The similarity between equation (1b) and Hamilton's (1964) rule,

$$
C<r B
$$

appears when we note that the left-hand sides of both equations (1b) and (2) represent a cost to the actor, while the right-hand sides represent the benefits to the recipient, weighted by the coefficient of relatedness. Hamilton's rule often takes the form of an inequality as in equation (2) because altruistic behaviors are often modeled as discrete alternatives: fixed actions with predefined costs and benefits are considered, and then the conditions under which these actions can spread are derived. Here we assume, instead, that costs and benefits are continuous functions of some amount of investment in altruism, and then we derive the equilibrium value of investment (hence the equality in eq. [1b]). This, of course, implies that assumptions are to be made on two functions rather than two values. In order to converge toward inner equilibrium values, we have to assume that marginal benefits exceed marginal costs at low investment values, while the reverse occurs at high investment values (as necessarily happens if fitness is a saturating function of the resource exchanged). This can be modeled, for example, by two power functions with different coefficients (fig. 1) in which costs increase first slowly, then rapidly with investments, while returns in benefits are first high and then diminish.

A second comment has to be made on the way we formalize altruism. Wilson et al. (1992) and Taylor (1992) assumed a "diffuse" altruism, in which altruistic actions benefit the whole neighborhood, including the actor itself. Corresponding biological situations may comprise the building or defense of a common nest, or cooperative hunting of large prey. In our case, because we want to model situations in which altruism is conditional to the status of recipients (familiarity-based recognition), we have to assume pairwise interactions. An individual engages in a pairwise relation with a suitable candidate, and this action brings benefits $B$ to the recipient and costs $C$ to himself. In other words, the actor gains no share of the benefit: its action is purely altruistic. Corresponding biological situations may include the share of a territory or any other breeding resource by its owner. Formally, we assume that every individual gets a basic fecundity value $m_{o}$, from which $C$ must be retrieved if this individual concedes an altruistic action, and to which $B$ must be added if this individual benefits from such an altruistic action from a social partner $(C$ and $B$ being functions of the amount of altruism, $p$ ). We show in appendix $A$ that this formalization does not change the conclusions of Wilson et al. (1992) and Taylor (1992): the inclusive benefits of indiscriminate altruism are exactly cancelled out by the additional kin competition.

A few simplifying assumptions were also made for con-

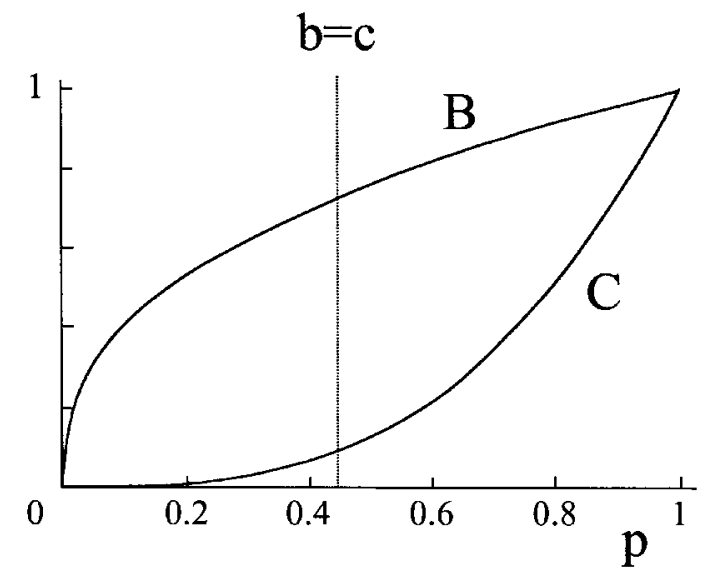

Figure 1: Benefits $(B)$ and costs $(C)$ modeled as power functions of altruistic investment $(p)$. In all simulations presented here, the functions $B=p^{0.4}$ and $C=p^{3}$ were used. A vertical dashed line is drawn where marginal costs and benefits equalize, that is, where curves have the same slope (at $p=0.455$ for our parameter values). 
venience. We consider an infinite-island population model with random dispersal, so that relatedness among patches drops to 0 and immigrants find no relatives among their new patch mates. Dispersal is followed by competition for breeding spots, then by social interactions and reproduction. We also assume nonoverlapping generations and asexual reproduction, which facilitates the dynamic accounting of relatedness.

Because altruistic interactions may be conditional to the dispersal status of actors and recipients, the fecundity of a locally born female may differ from that of an immigrant, which affects the dynamics of relatedness. The equilibrium value is given by (app. B)

$$
r=\frac{k m_{l}^{2}+\tilde{k} m_{i}^{2}}{N m_{p}^{2}-k^{2} m_{l}^{2}(N-1)},
$$

where $N$ is the number of females breeding in the patch, $k$ the probability that a breeding female was born locally, $\tilde{k}=1-k$ the probability that a breeding female was an immigrant, $m_{l}$ the fecundity of a locally born female, $m_{i}$ that of an immigrant female, and $m_{p}=k m_{l}+\tilde{k} m_{i}$ that of an average female. The main parameters used throughout our model are otherwise defined in appendix C.

\section{Analyses}

\section{Familiarity-Based Recognition}

In the first situation investigated, social interactions take place only among residents because associative learning allows actors to individually recognize relatives from immigrants and only behave altruistically when encounter occurs with a coresident. The fitness function is calculated by noting the following two factors. First, the focal female stays philopatric with probability $\tilde{x}_{i j}$, in which case she pays the cost $C_{p_{i j}}$ with probability $k_{x_{j}}$ (probability to meet a relative), and receives the benefit $B_{p_{j}}$ from a relative with the same probability. Competition for breeding spots occurs among $m_{p_{j}} \tilde{x}_{j}+m_{p} x s$ other females, where $m_{p_{j}}=$ $m_{o}+k_{x}^{2}\left(B_{p_{j}}-C_{p_{j}}\right)$ is the productivity of the parental generation on the focal patch and $m_{p}=m_{o}+k_{x}^{2}\left(B_{p}-C_{p}\right)$ that on other patches. And second, in the case the focal female disperses, she survives (or reaches reproductive status) with probability $s$, competes with $m_{p}(\tilde{x}+x s)$ unrelated females for a breeding patch, and is not involved in any social interaction. Her direct fitness is thus written

$$
W=\tilde{x}_{i j} \frac{m_{o}+k_{x_{j}}\left(B_{p_{j}}-C_{p_{i j}}\right)}{m_{p_{j}} \tilde{x}_{j}+m_{p} x s}+x_{i j} s \frac{m_{o}}{m_{p}(\tilde{x}+x s)} .
$$

Note that residents are fitter than immigrants (as long as $B>C$ ) since the latter do not benefit from altruistic interactions.

ES (Evolutionarily Stable) Altruism. The direct selective pressure on altruistic investments is obtained by deriving equation (4a) with respect to $p_{i j}$, and the indirect one, by deriving it with respect to $p_{j}$. Since selective pressures are derived only as a way to find ESSs, we will formulate them thereafter while assuming $p_{i j}=p_{j}=p$ and $x_{i j}=x_{j}=x$, which is always true at the equilibrium and considerably simplifies notations. Hence,

$$
\begin{aligned}
& \frac{\partial W}{\partial p_{i j}}=-\frac{k_{x}^{2} c_{p}}{m_{p}}, \\
& \frac{\partial W}{\partial p_{j}}=\frac{k_{x}^{2} b_{p}-k_{x}^{4}\left(b_{p}-c_{p}\right) \varphi}{m_{p}},
\end{aligned}
$$

where $c_{p}=d C_{p} / d p$ is the marginal increase in costs with altruistic investments, $b_{p}=d B_{p} / d p$ is the marginal increase in benefits, and $\varphi=\left[m_{o}+k_{x}\left(B_{p_{j}}-C_{p_{j}}\right)\right] /\left[m_{o}+k_{x}^{2}\left(B_{p_{j}}-\right.\right.$ $\left.\left.C_{p_{j}}\right)\right] \geq 1$ is the fecundity of a resident, relative to that of an average female. The complete selective pressure is given by $\partial W / \partial p_{i j}+r\left(\partial W / \partial p_{j}\right)$, and the ESS is found by setting this pressure to 0 . Hence,

$$
c_{p}=r\left[b_{p}-k_{x}^{2}\left(b_{p}-c_{p}\right) \varphi\right] .
$$

The left-hand side represents the marginal costs of altruism, and the right-hand side, its marginal benefits weighted by the kin coefficient of relatedness between actor and recipient. The net benefits consist of additional fecundity $\left(b_{p}\right)$ devaluated by the effects of additional kin competition. The latter increases with the relative fecundity of residents $(\varphi)$ as well as with philopatry $(k)$. However, as relatedness (r) also increases with philopatry (eq. [3]), the two effects cancel out in such a way that the equilibrium altruism appears largely unaffected by dispersal (fig. $2 A$ ). Note also that the selective pressure on altruism vanishes as $k$ (and thereby $r$; eq. [3]) tends to unity: condition (4d) is then met whatever the value of $p$, which means that altruism becomes a neutral character in absence of dispersal.

ES Dispersal. The direct selective pressure on dispersal is obtained as the partial derivative of equation (4a):

$$
\frac{\partial W}{\partial x_{i j}}=\frac{-\tilde{s} m_{o}-k_{x}\left(B_{p}-C_{p}\right)}{m_{p}(\tilde{x}+x s)} .
$$

This derivative contains two negative terms, showing that emigrants lose both in terms of survival (mortality costs of dispersal) and fecundity (they do not benefit from altruistic interactions). This combined effect brings strong 

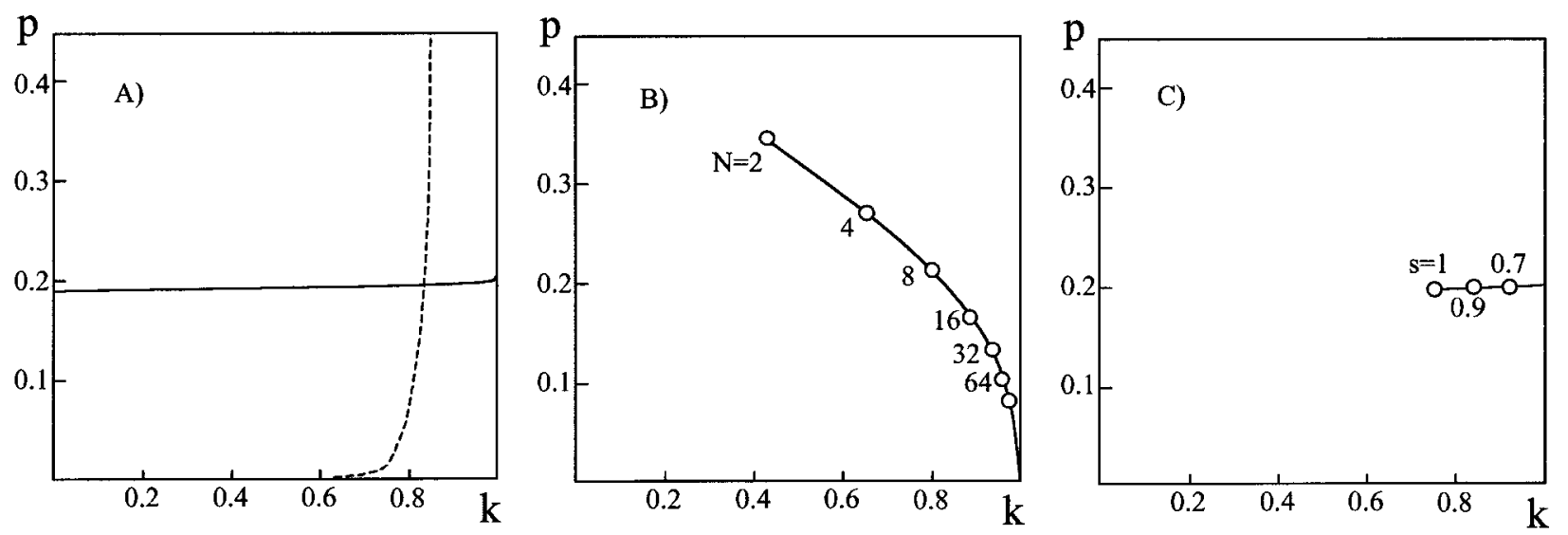

Figure 2: ES philopatry $(k)$ and altruism $(p)$ patterns under associative learning. A, Altruism shows extremely low dependence on dispersal (solid line), while philopatry increases markedly and monotonically with altruism (dashed line), reaching a maximum when the benefits of altruism are also at a maximum $(p=0.455)$. Results displayed for $N=10$ and $s=0.9 . B$, Varying patch size induces a strong negative correlation between altruism and philopatry, with maximal altruism and dispersal at low population sizes (due to strong kinship). Results displayed for $s=0.9$. $C$, Varying dispersal costs induce an increase in philopatry, while individual altruistic investments remain unaffected. Philopatry and altruism show positive values even in the absence of costs $(s=1)$. Results displayed for $N=10$.

direct incentives to philopatry. The only force behind dispersal stems from the indirect selective pressure:

$$
\frac{\partial W}{\partial x_{j}}=\frac{k_{x}\left[m_{o}+k_{x}\left(B_{p}-C_{p}\right)\right]-\tilde{k}_{x} k_{x}\left(B_{p}-C_{p}\right)}{m_{p}(\tilde{x}+x s)}
$$

which shows one positive component: the first term in the right-hand side corresponds to the value of the breeding opportunity left behind, weighted by the probability that this opportunity is seized by a relative (these are the benefits of avoiding kin competition). There is, however, also one negative component (second term in the right-hand side), corresponding to the probability that this opportunity is taken by an immigrant. In this case, the relatives of the disperser will lose one possibility to interact (and with it the associated net benefit $B_{p}-C_{p}$ ). The complete selective pressure vanishes when

$$
\begin{aligned}
& \tilde{s} m_{o}+k_{x}\left(B_{p}-C_{p}\right)= \\
& r k_{x}\left[m_{o}+\left(k_{x}-\tilde{k}_{x}\right)\left(B_{p}-C_{p}\right)\right]
\end{aligned}
$$

or, equivalently, when

$$
\tilde{s}+\rho=r[k(1+\rho)-\tilde{k} \rho]
$$

where $\rho=\left(m_{l}-m_{i}\right) / m_{i}=\left[k_{x}\left(B_{p}-C_{p}\right)\right] / m_{o}$ represents the relative fecundity advantage of residents over immigrants. This result can also be interpreted in light of Hamilton's equation (dispersal as a means to avoid kin competition can be considered an altruistic act). The left-hand side of equation (4h) is the cost of the action to the actor (which sacrifices some of its own survival probability and fecundity by dispersing), while the right-hand side represents the benefits to recipients, weighted by the kin coefficient of relatedness between actor and recipient. Figure $2 \mathrm{~A}$ shows that philopatry increases strongly and monotonically with altruism, up to a maximum reached when marginal costs and benefits equalize.

Figure 2 (fig. $2 B, 2 C$ ) displays the joint equilibrium under various $N$ and $s$ values. As this figure shows, positive altruism and philopatry values are selected even in absence of costs to dispersal. The benefits of altruism overweigh the costs of kin competition and allow strong kin structures to emerge. Philopatry is a consequence of social interactions not a prerequisite for them to evolve.

\section{Spatially Based Discrimination}

We now analyze the case where investment is triggered by spatial cues, being expressed only when the focal individual is resident but independently of the recipient's dispersal status. The cost $C_{p_{i j}}$ is thus paid when the actor is resident (and whatever its partner) but not when the actor is an immigrant into another patch. Reciprocally, the focal female will benefit with probability $k_{x_{i}}$ from the altruism of related residents if she remains local and with probability $k_{x}$ from the altruism of unrelated residents if she moves. Hence, 

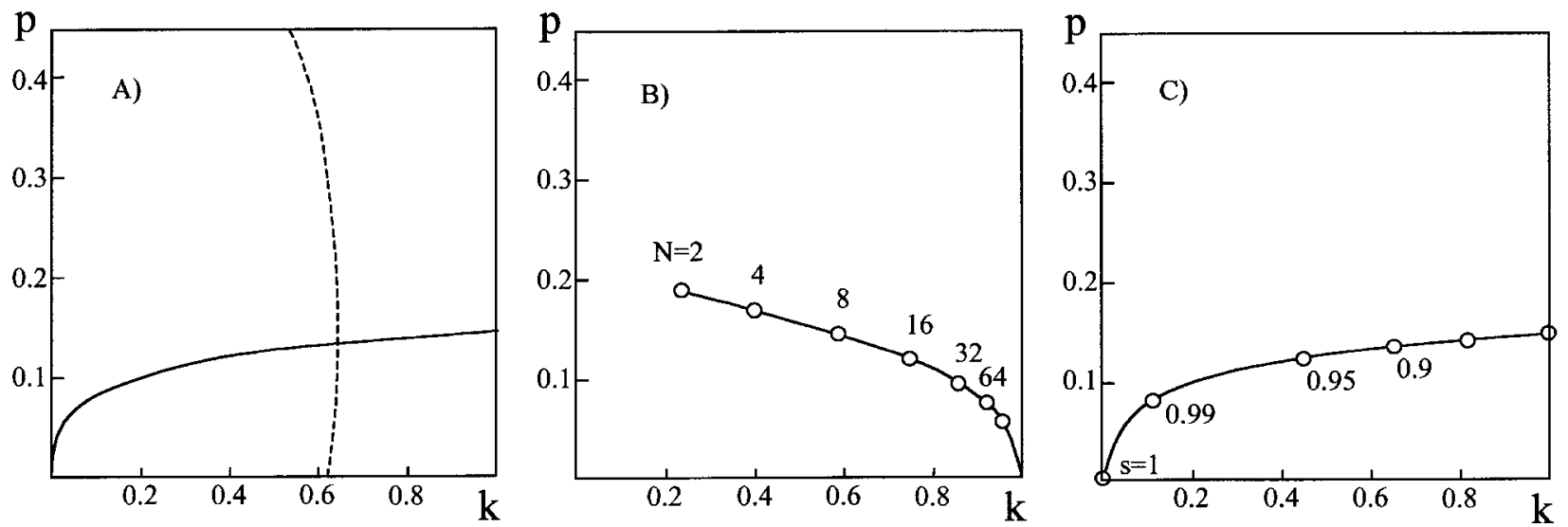

Figure 3: ES philopatry $(k)$ and altruism $(p)$ patterns under spatially based discrimination. A, Altruism increases sharply with philopatry (solid line), while philopatry first increases, then decreases with altruism (dashed line). Results displayed for $N=10$ and $s=0.9$. B, Varying patch size induces a negative correlation between dispersal and altruism. The latter's maximal value is only about half that found under associative learning. Results displayed for $s=0.9$. C, Varying dispersal costs increases both philopatry and altruism, which induces a positive correlation between these traits. Both traits vanish in absence of mortality costs $(s=1)$. Results displayed for $N=10$.

$$
W=\tilde{x}_{i j} \frac{m_{o}-C_{p_{i j}}+k_{x_{j}} B_{p_{j}}}{m_{p_{j}} \tilde{x}_{j}+m_{p} x s}+x_{i j} s \frac{m_{o}+k_{x} B_{p}}{m_{p}(\tilde{x}+x s)} .
$$

The average productivity of the parental generation in patch $j$ is now $m_{p_{j}}=m_{o}+k_{x}\left(B_{p_{j}}-C_{p_{j}}\right)$, and that in average patches, $m_{p}=m_{o}+k_{x}\left(B_{p}-C_{p}\right)$ because only a proportion $k_{x}$ of the breeding females are resident and thus display altruism. It is important to note that in such a situation the fitness of an immigrant exceeds that of a resident: both benefit from the altruism of residents around, but only residents pay the costs. This is bound to have strong consequences on both altruism and dispersal ES values.

ES Altruism. Deriving equation (5a) with respect to $p_{i j}$ provides

$$
\frac{\partial W}{\partial p_{i j}}=\frac{-c_{p} k_{x}}{m_{p}}
$$

while deriving it with respect to $p_{j}$ gives

$$
\frac{\partial W}{\partial p_{j}}=\frac{k_{x}^{2}\left[b_{p}-k_{x} \varphi\left(b_{p}-c_{p}\right)\right]}{m_{p}}
$$

where $\varphi=\left(m_{o}+k B-C\right) /\left[m_{o}+k(B-C)\right] \leq 1$ is the fecundity of a resident relative to that of an average breeding female.

The complete selective pressure on altruism $\left(\partial W / \partial p_{i j}+\right.$ $\left.r\left[\partial W / \partial p_{j}\right]\right)$ vanishes when

$$
c_{p}=r\left[k_{x} b_{p}-k_{x}^{2}\left(b_{p}-c_{p}\right) \varphi\right]
$$

Relative to the case with perfect discrimination (eq. [4d]), the benefits through increased fecundity are weighted by a factor $k_{x}$ because only residents behave altruistically. As a result, the optimal investment now depends strongly on dispersal patterns (fig. 3A). While altruism can evolve to some extent if philopatry is high, it progressively vanishes as dispersal increases: altruistic investments are wasted if they benefit only unrelated immigrants.

ES Dispersal. The direct selective pressure on dispersal is given by

$$
\frac{\partial W}{\partial x_{i j}}=\frac{C_{p}-\tilde{s}\left(m_{o}+k_{x} B_{p}\right)}{m_{p}(\tilde{x}+x s)} .
$$

As can be seen, the cost of altruism becomes there an incentive for dispersal (because immigrants do not have to pay it), while the second term in the right-hand side numerator, stemming from the cost of dispersal, opposes this incentive. The indirect pressure is obtained from

$$
\frac{\partial W}{\partial x_{j}}=\frac{k_{x}\left(m_{o}+k_{x} B_{p}-C_{p}\right)-\tilde{k}_{x} k_{x} B_{p}}{m_{p}(\tilde{x}+x s)} .
$$

This last expression also comprises one positive and one negative term. The first one corresponds to the value of the breeding opportunity left behind by an emigrant, weighted 
by the probability that this opportunity is seized by a relative. The negative term expresses the possibility that this opportunity is taken by an immigrant, in which case relatives will lose a benefit $B$ (as immigrants invest nothing).

This situation thus contrasts with the preceding cases in which direct selective pressures on dispersal were always negative. Here, individual selection comprises a positive component and kin selection a negative one: dispersal may thus become a selfish action (avoiding to pay the costs of altruism) while philopatry may become an altruistic act, selected through its benefits on relatives. The complete selective pressure vanishes when

$$
\tilde{s}\left(m_{o}+k_{x} B_{p}\right)-C_{p}=r k_{x}\left[m_{o}+\left(k_{x}-\tilde{k}_{x}\right) B_{p}-C_{p}\right] .
$$

Optimal dispersal now depends nonmonotonically on altruism (fig. $3 \mathrm{~A}$ ), first increasing, then decreasing with it. This complex behavior results from the interplay of opposing forces evidenced above. As the burden of altruism costs becomes too heavy, individual selection promotes dispersal.

Figure 3 (fig. 3B, 3C) shows the joint equilibrium for different $N$ and $s$ values. An important point is that as dispersal costs vanish dispersal becomes complete (to avoid both kin competition and the cost of altruism), and this, in turn, makes altruism vanish. In other words, altruism needs dispersal costs in order to evolve. The emergence of sociality is thus conditional to the previous establishment of philopatry through ecological constraints.

\section{Discussion}

\section{Kin Discrimination and Ecological Constraints}

Hamilton's rule has the potential to initiate a positive feedback between philopatry and altruism. On the one hand, philopatry creates kin structure, which should favor altruistic behavior. On the other hand, the expected benefits of altruism among kin should favor philopatry. However, this self-reinforcing loop may be blocked by kin competition, which by suppressing all benefits arising from altruistic kin interactions, prevents the evolution of social structures. This is why the interplay between altruism and dispersal in any species is modulated by its kin-discrimination abilities, which play a major role in disentangling social and competitive neighborhoods (Queller 1992, 1994).

The point must be made that the two kin-discrimination mechanisms investigated here ultimately rely on spatial cues. The only difference is that recognition is considered as "spatially based" if spatial cues have to be used at the very moment of providing altruism. By contrast, associative learning allows organisms to build a mental association between spatial cues and the individual characteristics of neighbors at a time when relatedness and proximity present the maximal correlation. This association (or imprinting) allows altruism to be subsequently allocated correctly, at times at which correlation is normally much weaker. Thus, the benefits of associative learning stem basically from a temporal decoupling between spatial association and altruistic investment. This decoupling obviously makes big differences if dispersal takes place in the meanwhile.

Differences are best seen when comparing figures $2 A$ and $3 A$. Under associative learning (fig. $2 A$ ), altruism shows extremely low dependence on dispersal, which contrasts with the strong dependence observed under spatially based discrimination (fig. 3A), in which altruism progressively vanishes as dispersal increases. Mathematically, this is explained by the fact that philopatry $\left(k_{x}\right)$ weights the benefits of altruism $\left(r b_{p}\right)$ in equation (5d) not in equation (4d). More intuitively, this arises because, under spatially based discrimination, altruistic investment is blind, being spent mostly on unrelated immigrants when dispersal is high. By contrast, associative learning allows altruism to be directed only toward relatives, even when their relative density becomes vanishingly small.

Dispersal patterns also differ. Under associative learning (fig. 2A), philopatry is high and increases monotonically with the benefits of altruism $\left(B_{p}-C_{p}\right)$, while under spatially based discrimination (fig. $3 A$ ), a complex behavior emerges: philopatry first increases, then decreases with altruism. This results from the selfish component of dispersal characteristic of this situation: as immigrants benefit from the altruism of residents without having to pay its costs, their fitness exceeds that of residents, so that high altruism values select for high dispersal. This situation differs qualitatively from the previous one in which individual selection always selects against dispersal. As a matter of fact, that philopatry may exist at all under spatial discrimination may seem counterintuitive at first view since residents have a lower fecundity than immigrants. The point is that the constraints on dispersal lower the fitness expectancy of emigrants. Philopatry thus evolves only insofar as the fitness costs of dispersal exceed the additional fecundity benefits of immigrants. The frequency dependence of these benefits (the more immigrants, the less they benefit from the altruism of residents) make an inner equilibrium possible.

As a result, dispersal costs constitute a prerequisite for altruism to evolve under spatially based discrimination (fig. $3 C$ ). In absence of dispersal costs $(s=1)$, all individuals disperse in order to avoid kin competition, so that altruism cannot evolve. As costs increase, some individuals stay home, from which social interactions may emerge as the best of a bad job. It appears, thus, that the dynamic accounting of dispersal and relatedness makes the conditions for the evolution of altruism more complex than previously thought. According to Queller (1992, 1994), a 
necessary condition for altruism to emerge is that partners be more related in social than in economic interactions. This condition seems sufficient as long as the dispersal rate is fixed but not if dispersal is allowed to coevolve with altruism (as in this approach). Our results indeed show that under spatially based discrimination, a further condition is required, namely, the existence of ecological constraints to dispersal. In the absence of such constraints, social life disappears. This is precisely the outcome envisaged by the "ecological-constraint" model (Emlen 1982; Jarvis et al. 1994; Spinks et al. 2000).

The situation differs drastically when associative learning allows perfect discrimination among potential recipients. A strong philopatry and a high altruism level are expected even in the absence of dispersal costs (fig. 2C). Increasing these costs enhances philopatry, with no detectable effect on altruism level. The benefits of altruism largely outweigh the costs of additional kin competition and thereby provide strong incentives for individuals to stay home. Philopatry is thus enforced by sociality, which is precisely the outcome envisaged by the "benefits-ofphilopatry" model (Stacey and Ligon 1991).

\section{Empirical Avenues}

Our approach thus points to a direct connection between two fields of behavioral ecology considered up to now to be relatively independent: on the one hand, the debate over the role of extrinsic constraints versus intrinsic benefits in the evolution of social interactions; on the other hand, the study of the kin-discrimination mechanisms on which these interactions rely. Because of this relative independence, empirical data that might falsify or corroborate our model seem pretty scarce. From the review of Komdeur and Hatchwell (1999, p. 238) on birds, for instance, "there have been no published experimental studies of kin recognition among cooperative species, and little attention has been paid to the actual cues used by helpers when making helping decisions." Below, we suggest a few predictions and avenues of research that may help to test our model.

First, we expect the role of constraints to depend on the cognitive ability and life-history characteristics of the species under study. Associative learning requires higher cognitive abilities from the actors (namely, the capacity first to associate spatial cues and individual characteristics and then to memorize these associations up to the time when altruistic investments are to be made). The requirement of a period of familiarization with relatives is more likely to be satisfied in species with extended parental care. Furthermore, recognizing specific individuals may be relatively straightforward in small families but presents greater cognitive challenges in larger groups. Our analysis thus predicts that ecological constraints are more likely to affect social evolution in species in which restricted cognitive abilities, large group size, and/or limited parental care impose kin discrimination to rely on spatial cues, while the benefit-ofphilopatry model may prevail in those species (like many birds; Komdeur and Hatchwell 1999) in which high cognitive capacities, small family size, and extended parental care may allow associative learning to perform a better kin discrimination.

The fact that constraints have often been invoked in birds as a main cause of sociality (e.g., Koenig and Pitelka 1981; Marzluff and Balda 1990; Emlen 1991; Koenig et al. 1992; Hatchwell and Komdeur 2000) may at first view seem to oppose our prediction. However, the social parameter affected by ecological constraints in birds is not the amount of altruistic investment conceded by a philopatric individual (as expected under spatially based discrimination) but the probability that individuals will stay philopatric (and thus help). This is precisely the pattern expected under associative learning (fig. 2C): a proportion of individuals stay and help even in absence of constraints (as documented by, e.g., Stacey and Ligon 1991), and this proportion increases with constraints (as documented by, e.g., Komdeur 1992). An important distinction thus has to be made between individual- versus population-level amount of helping since, as we show, they may evolve independently. The former is a measure of individual investment in an altruistic interaction, and the latter, a measure of the proportion of individuals that stay home and interact altruistically. Independent quantification of both measures should provide insights into the conditions prevailing in the evolution of the specific interactions under study and should actually allow a test of our model, provided the kin-recognition mechanisms underlying these interactions are also documented.

The importance of constraints has also been largely advocated in Bathyergidae (naked mole rats and relatives). Social structures vary with ecological constraints and dispersal costs, both within and among species (Jarvis and Bennett 1991; Jarvis et al. 1994; Spinks et al. 2000). There is strong correlational evidence that both the number of individuals remaining home and their individual level of investment (social integration) increases with constraints (Jarvis et al. 1994). From our study, this points to low kinrecognition abilities, presumably not individually based, an expectation consistent with the fact that eusocial Bathyergidae live in large groups (up to 300 individuals in naked mole rats) and (except for reproductives) are very short lived (Jarvis et al. 1994). Existing data indeed suggest that kin recognition in mole rats is not individually based but relies instead on the specific odor of local colonies, rapidly gained by immigrants if they succeed in entering a colony (O'riain and Jarvis 1997).

Spatially based recognition may actually prevail even in vertebrates with high cognitive abilities whenever invest- 
ments have to be provided at the moment of maximal correlation between relatedness and proximity. Classical situations include mother-offspring interactions in mammals and nest feeding in birds (Waldman 1987; Komdeur and Hatchwell 1999). Parent birds feed whatever hatchling they find in their nest, a situation largely exploited by parasites (both conspecifics and allospecifics). In this respect, it is relevant to note that the level of paternal investment by cuckolded male birds often increases with their confidence of paternity (Davies et al. 1992; Hartley et al. 1995) in a way quite similar to what is expected from altruism under spatially based discrimination (fig. $3 A$ ). Males have no other cues than spatial ones to recognize their offspring, so their level of investment should decrease as the number of "immigrants” (eggs fertilized through extra-pair copulations) increases. We similarly expect that in the absence of constraints limiting the amount of extra-pair copulations, the whole system (paternal investment) would collapse.

Associative learning may develop after birth throughout the feeding period so that presumed offspring will be considered as such long after fledging or weaning times. This observation points to the fact that, in contrast with the assumptions of this model, associative learning does not warrant perfect correlation between altruism and relatedness. Imperfect correlation at the time of association, or imperfect memory at the time of allocation, are bound to affect our predictions. We expect in such a case intermediate outcomes, closer to the spatially based expectations if correlation at the time of association, or memory at the time of allocation, is bad.

The strength of social structures has also been suggested to correlate with environmental harshness, dispersal costs, and habitat saturation in social insects (Hölldobler and Wilson 1977; Pamilo 1981; Pamilo and Rosengren 1984; Keller 1995). Experimental manipulation of nesting opportunities has been shown to affect social structure in hymenopteran species in which breeding opportunities limit the fitness of dispersers. Polygyny in the ant Leptothorax longispinosus drops as more nest sites are made available (Herbers 1986). The number of helpers in the stenogastrine wasp Liostenogaster flavolineata decreases as more nests are provided (Field et al. 1998). Insects displaying low and variable levels of sociality might indeed constitute a good biological material to test our expectations, as discrimination mechanisms apparently show considerable diversity among species (Crozier and Pamilo 1996). The best empirical evidence should come from manipulating constraints in species that rely on different mechanisms and then monitoring both philopatry and social integration. Species using a spatially based discrimination (as might be the case in some polistine wasps; Waldman 1987) are expected to display complete dispersal in absence of constraints; increasing constraints should enhance both philopatry and social integration (levels of individual investments). By contrast, species using a familiarity-based discrimination are expected to display philopatry and sociality even in the absence of dispersal costs; constraints should only enhance philopatry not the level of individual investments. Interestingly, this seemed to be the outcome of the experiments on stenogastrine wasps mentioned above (Field et al. 1998): a significant proportion of females remained as helpers even when the immediate surroundings were experimentally saturated with opportunities for independent breeding. This suggests good kin-discrimination abilities, which is consistent with the fact that small group sizes in this species (one to four females) should enable individual recognition.

\section{Model Limitations}

How far do our results depend on the several assumptions underlying the model? Those made about the shapes of the functions delineating the costs and benefits of altruism were quite specific (fig. 1), but we also used power functions with different parameter values without any qualitative change in the conclusions (N. Perrin and L. Lehmann, unpublished data). We actually expect a similar behavior from any set of functions in which marginal costs increase monotonically while marginal benefits decrease monotonically.

Our infinite-island-model assumptions are unlikely to affect qualitatively our conclusions either. In finite-island models, immigrants still have zero relatedness (or nearly so) with residents. If the island assumption is dropped (e.g., gene flow decreases with distance), then residents are somewhat related to immigrants (as long as the whole population is taken as reference) but less so than to other residents, so that predictions should not differ qualitatively.

The habitat-saturation assumption is certainly not always verified in practice (if only for demographic stochasticity). Unsaturated environments are expected to lower kin-competition pressures and, thereby, to increase the overall philopatry and altruism levels. This should also soften the contrasts among the kin-discrimination mechanisms investigated here. A similar comment can be made about the timing of events: if social interactions are allowed to occur before dispersal and reproduction (as might be the case, e.g., for insect larvae building a common defense against predators) in such a way that the benefits of sociality can be exported, then kin competition will no longer counteract the emergence of altruistic behaviors.

Finally, the point must be made that many of the social species mentioned above have overlapping generations (this trait being actually part of the definition of eusociality), while our model assumes discrete generations. Overlap is likely to affect the dynamics of relatedness and, 
more importantly, to introduce asymmetries in pairwise interactions, in terms of competitiveness, costs and benefits of altruism, reproductive value, and access to information (young females having no information about the dispersing status of older partners). Furthermore, formalization efforts aimed at integrating the complexities stemming from generational overlap are beyond the scope of this article but might prove to be useful before the ideas developed here can be properly and rigorously tested.

\section{Acknowledgments}

Several vine growers from the Côtes-du-Rhône region (especially those of Rasteau and Gigondas) contributed significantly to the elaboration and formalization of our ideas. We want to thank colleagues from our department (especially F. Balloux, M. Chapuisat, P. Christe, T. Giraud, J. Goudet, L. Keller, J. Pedersen, E. Petit, and M. Reuter) and two anonymous referees for fruitful discussions and/ or useful comments on a previous version of this manuscript. This work benefited from the financial support of the Swiss National Science Foundation (grants 3138762.93 and 31-59442.99 to N.P.).

\section{APPENDIX A}

\section{The Case of Indiscriminate Altruism}

Here we show that our specific formalization of altruism (continuous settings, pairwise interactions, and coevolution with dispersal) does not change the conclusions of Wilson et al. (1992) and Taylor (1992): the inclusive benefits of indiscriminate altruism are exactly cancelled out by the additional kin competition generated. Indeed, in absence of discrimination, the focal female pays a cost $C_{P_{i j}}$ in every situation. If she disperses and succeeds in getting established, she will benefit from the unconditional altruism of unrelated females $\left(B_{p}\right)$. If she decides to stay as a resident, she will benefit from interactions with either related or unrelated females, with probabilities that depend on dispersal rate

$$
\begin{aligned}
W= & \tilde{x}_{i j} \frac{m_{o}-C_{p_{i j}}+k_{x_{j}} B_{p_{j}}+\tilde{k}_{x_{j}} B_{p}}{m_{p_{j}} \tilde{x}_{j}+m_{p} x s} \\
& +x_{i j} s \frac{m_{o}-C_{p_{i j}}+B_{p}}{m_{p}(\tilde{x}+x s)} .
\end{aligned}
$$

The productivity from the focal patch is measured as $m_{p j}=m_{o}+B_{p_{j}}-C_{p j}$ and that of other patches, $m_{p}=$
$m_{o}+B_{p}-C_{p}$ since all females from the parental generation interacted altruistically.

\section{ES Altruism}

The direct selective pressure on altruism is obtained as the derivative of equation (A1a) with respect to $p_{i j}$ :

$$
\frac{\partial W}{\partial p_{i j}}=-\frac{c_{p}}{m_{p}},
$$

while indirect pressure is obtained by

$$
\frac{\partial W}{\partial p_{j}}=\frac{k_{x}^{2} c_{p}}{m_{p}} .
$$

As can be seen, the benefits of altruism do not appear in this equation because they are exactly cancelled out by the increase in competition among kin. The complete selective pressure is given by $\partial W / \partial p_{i j}+r\left(\partial W / \partial p_{j}\right)$, which vanishes when

$$
c_{p}=0,
$$

that is, when the costs of investments are at a minimum. In our formalization, this corresponds to no investment $(p=0)$. Note that the complete selective pressure vanishes as before (i.e., altruism becomes a neutral character) when $k=r=1$.

\section{ES Dispersal}

The direct selective pressure on dispersal is

$$
\frac{\partial W}{\partial x_{i j}}=\frac{s-1}{\tilde{x}+x s}
$$

while the indirect one is

$$
\frac{\partial W}{\partial x_{j}}=\frac{k_{x}}{\tilde{x}+x s} .
$$

Thus the inclusive-fitness pressure on dispersal, given by $\partial W / \partial x_{i j}+r\left(\partial W / \partial x_{j}\right)$, vanishes when

$$
\tilde{s}=r k_{x} \text {. }
$$

This result, identical to Frank (1986), shows that dispersal evolves here only through kin-competition avoidance and independently of the level of altruism. 


\section{APPENDIX B}

\section{Recurrence Equation for Relatedness}

Two randomly chosen offspring have relatedness 1 if they share the same mother and relatedness $r$ if their mothers differ but were both local (more precisely, they have, in this case, relatedness 1 with probability $r$ ). The probability that two randomly chosen offspring were born from one given local mother is $\left(m_{l} / N m_{p}\right)^{2}$, and there are $k N$ such mothers. Similarly, the probability that two randomly chosen offspring were born from one given immigrant mother is $\left(m_{i} / N m_{p}\right)^{2}$, and there are $\tilde{k} N$ such mothers. Finally, the probability that two offspring were born from local but distinct mothers is $k^{2} N(N-1)\left(m_{l} / N m_{p}\right)^{2}$. Collecting these terms provides the recurrence equation

$$
r^{\prime}=\frac{k m_{l}^{2}+\tilde{k} m_{i}^{2}}{N m_{p}^{2}}+\frac{k^{2}(N-1) m_{l}^{2}}{N m_{p}^{2}} r
$$

from which the equilibrium value is derived by setting $r^{\prime}=r$ :

$$
r=\frac{k m_{l}^{2}+\tilde{k} m_{i}^{2}}{N m_{p}^{2}-\left(k m_{l}\right)^{2}(N-1)} .
$$

Note that when the fitness of immigrant and resident females are equal, then equation (B2) reduces to the expression used by Taylor (1992) for asexually reproducing organisms:

$$
r=\frac{1}{N-k^{2}(N-1)}
$$

\begin{tabular}{|c|c|}
\hline Parameter & Definition \\
\hline$N$ & Number of breeding females \\
\hline$x_{i j}$ & $\begin{array}{l}\text { Dispersal probability of female } i \text { born in patch } j ; x_{j} \text { is the average for patch } j \text { and } x \text { the average for the whole } \\
\text { metapopulation }\end{array}$ \\
\hline$s$ & $\begin{array}{l}\text { Probability (relative to that of a resident) that a disperser reaches a breeding status; it might represent, for } \\
\text { example, survival during dispersal; } \tilde{s} \text { is thus the ecological constraint to dispersal (e.g., mortality cost) }\end{array}$ \\
\hline$k_{x}=\tilde{x}_{j} /\left(\tilde{x}_{j}+x s\right)$ & $\begin{array}{l}\text { Probability that a female randomly taken from patch } j \text { after dispersal is a resident; } \tilde{k}_{x} \text { is thus the effective } \\
\text { migration rate into patch } j\end{array}$ \\
\hline$p_{i j}$ & $\begin{array}{l}\text { Amount of investment by the focal individual in an altruistic act; } p_{j} \text { is the average investment of females } \\
\text { born on the same patch as well as that of the parental generation, which also bears relatedness } r \text { to the } \\
\text { focal individual }\end{array}$ \\
\hline$C_{p_{i j}}$ & Fecundity cost of altruism by the focal individual, an accelerating function of its investment $p_{i j}$ \\
\hline$B_{p_{j}}$ & Fecundity benefit to the recipient of an altruistic act, a decelerating function of investment \\
\hline$m_{o}$ & Fecundity by default, in the absence of any interaction \\
\hline$m_{i}$ & Fecundity of an immigrant female; further specification will depend on how altruism is expressed \\
\hline$m_{l} \quad \quad \quad$ & Fecundity of a locally born female; further specification will depend on how altruism is expressed \\
\hline & Average fecundity in patch $j$; the average for the whole metapopulation is $m_{p}$ \\
\hline
\end{tabular}

\section{APPENDIX C}

Table C1: Parameter definitions

Note: The parameters under selection (altruism and dispersal) have subscripts $i j$ if they refer to a phenotypic action under control of the focal individual and $j$ when referring to a phenotypic action controlled by social partners bearing relatedness $r$ to the focal individual. Absence of a subscript refers to the average value for the whole metapopulation. Tilde indicates complements to unity (e.g., $\tilde{x}_{j}=1-x_{j}$ ).

\section{Literature Cited}

Brown, L. H. 1974. Alternate routes to sociality in jays - with a theory for the evolution of altruism and communal breeding. American Zoologist 14:63-80.

Crozier, R. H., and P. Pamilo. 1996. Evolution of social insect colonies. Oxford University Press, Oxford.

Davies, N. B., B. J. Hatchwell, T. Robson, and T. Burke. 1992. Paternity and parental effort in dunnocks Prunella modularis: how good are male chick-feeding rules? Animal Behaviour 43:729-745.

Emlen, S. T. 1982. The evolution of helping. I. An ecological constraints model. American Naturalist 119:29-39.

. 1991. Evolution of cooperative breeding in birds and mammals. Pages 301-337 in J. R. Krebs and N. B. Davies, eds. Behavioural ecology: an evolutionary approach. Blackwell Scientific, Oxford. 1994. Benefits, constraints, and the evolution of 
the family. Trends in Ecology \& Evolution 9:282-285.

Ferrière, R., and J.-F. Le Gaillard. 2001. Invasion fitness and adaptive dynamics in spatial population models. Pages 57-79 in J. Clobert, E. Danchin, A. A. Dhondt, and J. D. Nichols, eds. Dispersal. Oxford University Press, Oxford.

Field, J., W. Foster, G. Shreeves, and S. Sumner. 1998. Ecological constraints on independent nesting in facultatively eusocial hover wasps. Proceedings of the Royal Society of London B, Biological Sciences 265:973-977.

Frank, S. A. 1986. Dispersal polymorphism in subdivided populations. Journal of Theoretical Biology 122:303-309.

. 1997. Multivariate analysis of correlated selection and kin selection, with an ESS maximization method. Journal of Theoretical Biology 189:307-316.

- 1998. Foundations of social evolution. Princeton University Press, Princeton, N.J.

Gaston, A. J. 1978. The evolution of group territorial behavior and cooperative breeding. American Naturalist 112:1091-1100.

Hamilton, W. D. 1964. The genetical evolution of social behaviour. I/II. Journal of Theoretical Biology 7:1-52.

Hamilton, W. D., and R. M. May. 1977. Dispersal in stable habitats. Nature 269:578-581.

Hartley, I. R., N. B. Davies, B. J. Hatchwell, A. Desrochers, D. Nebel, and T. Burke. 1995. The polygynandrous mating system of the alpine accentor, Prunella collaris. II. Multiple paternity and parental effort. Animal Behaviour 49:789-803.

Hatchwell, B. J., and J. Komdeur. 2000. Ecological constraints, life-history traits and the evolution of cooperative breeding. Animal Behaviour 59:1079-1086.

Herbers, J. M. 1986. Nest site limitation and facultative polygyny in the ant Leptothorax longispinosus. Behavioral Ecology and Sociobiology 19:115-122.

Hölldobler, B., and E. O. Wilson. 1977. The number of queens: an important trait in ant evolution. Naturwissenschaften 64:8-15.

Jarvis, J. U. M., and N. C. Bennett. 1991. Ecology and behavior of the family Bathyergidae. Pages 66-96 in P. W. Sherman, J. U. M. Jarvis, and R. D. Alexander, eds. The biology of the naked mole-rat. Princeton University Press, Princeton, N.J.

Jarvis, J. U. M., M. J. O'riain, N. C. Bennett, and P. W. Sherman. 1994. Mammalian eusociality: a family affair. Trends in Ecology \& Evolution 9:47-51.

Keller, L. 1995. Social life: the paradox of multiple-queen colonies. Trends in Ecology \& Evolution 10:355-360.

Koenig, W. D., and F. A. Pitelka. 1981. Ecological factors and kin selection in the evolution of cooperative breeding in birds. Pages 262-280 in R. D. Alexander and D. W. Tinkle, eds. Natural selection and social behavior: recent research and new theory. Chiron, New York.

Koenig, W. D., F. A. Pitelka, W. J. Carmen, R. L. Mumme, and M. T. Stanback. 1992. The evolution of delayed dispersal in cooperative breeders. Quarterly Review of Biology 67:111-150.

Komdeur, J. 1992. Importance of habitat saturation and territory quality for evolution of cooperative breeding in the Seychelles warbler. Nature 358:493-495.

Komdeur, J., and B. J. Hatchwell. 1999. Kin recognition: function and mechanisms in avian societies. Trends in Ecology \& Evolution 14:237-241.

Marzluff, J. M., and R. P. Balda. 1990. Pinyon jays: making the best of a bad situation by helping. Pages 197-237 in P. B. Stacey and W. D. Koenig, eds. Cooperative breeding in birds: long-term studies of ecology and behavior. Cambridge University Press, Cambridge.

Mitteldorf, J., and D. S. Wilson. 2000. Population viscosity and the evolution of altruism. Journal of Theoretical Biology 204:481-496.

O'riain, M. J., and J. U. M. Jarvis. 1997. Colony member recognition and xenophobia in the naked mole rat. Animal Behaviour 53:487-498.

Pamilo, P. 1981. Genetic organization of Formica sanguinea populations. Behavioral Ecology and Sociobiology 9: 45-50.

Pamilo, P., and R. Rosengren. 1984. Evolution of nesting strategies of ants: genetic evidence from different population types of Formica ants. Biological Journal of the Linnean Society 21:331-348.

Queller, D. C. 1992. Does population viscosity promote kin selection? Trends in Ecology \& Evolution 7:322-324.

- 1994. Genetic relatedness in viscous populations. Evolutionary Ecology 8:70-73.

Spinks, A. C., J. U. M. Jarvis, and N. C. Bennett. 2000. Comparative patterns of philopatry and dispersal in two common mole-rat populations: implications for the evolution of mole-rat sociality. Journal of Animal Ecology 69:224-234.

Stacey, P. B., and J. D. Ligon. 1987. Territory quality and dispersal options in the acorn woodpecker and a challenge to the habitat-saturation model of cooperative breeding. American Naturalist 130:654-676.

- 1991. The benefits-of-philopatry hypothesis for the evolution of cooperative breeding: variation in territory quality and group size effect. American Naturalist 137:831-846.

Taylor, P. D. 1988. An inclusive-fitness model for dispersal of offspring. Journal of Theoretical Biology 140:363-378.

- 1992. Altruism in viscous populations - an inclusive fitness model. Evolutionary Ecology 6:352-356.

Taylor, P. D., and S. A. Frank. 1996. How to make a kin selection model. Journal of Theoretical Biology 180: 27-37.

Waldman, B. 1987. Mechanisms of kin recognition. Journal of Theoretical Biology 128:159-185. 
Wilson, D. S., G. B. Pollock, and L. A. Dugatkin. 1992. Can altruism evolve in purely viscous populations? Evolutionary Ecology 6:352-356.

Zack, S. 1990. Coupling delayed breeding with short-distance dispersal in cooperatively breeding birds. Ethology 86:265-286.
Zack, S., and J. D. Ligon. 1985. Cooperative breeding in Lanius shrikes. II. Maintenance of group-living in a nonsaturated habitat. Auk 102:766-773.

Associate Editor: Joan E. Strassmann 